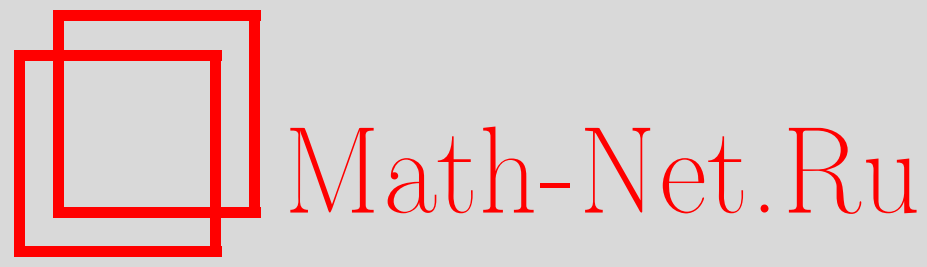

А. Л. Константинов, Инвариантное упорядочение на односвязном накрытии границы Шилова симметрической области, Функи. анализ и его прил., 2008, том 42, выпуск 1, 33-38

DOI: https://doi.org/10.4213/faa2888

Использование Общероссийского математического портала MathNet.Ru подразумевает, что вы прочитали и согласны с пользовательским соглашением

http://www . mathnet.ru/rus/agreement

Параметры загрузки:

IP : 54.81 .137 .203

26 апреля 2023 г., $17: 48: 14$

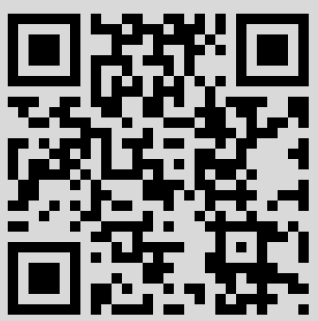


Функииональный анализ и его приложения

2008, т. 42, вып. 1, с. 33-38

УДК 512.816.4

\title{
Инвариантное упорядочение на односвязном накрытии границы Шилова симметрической области
}

\author{
(c) 2008. А. Л. КонстАнтинов
}

\section{§1. Введение}

Пусть $G$ - эрмитова простая группа Ли трубчатого типа. Граница Шилова симметрической области $D=G / K$ имеет вид $G / P$, где $P$ - такая связная максимальная параболическая подгруппа группы $G$, что ее унипотентный радикал абелев. В этой статье мы рассмотрим вопрос существования инвариантного упорядочения на односвязном накрытии пространства $G / P$, индуцированного инвариантным упорядочением на односвязном накрытии группы $G$.

В простейшем случае $D=\mathrm{SU}(1,1) / \mathrm{S}(\mathrm{U}(1) \times \mathrm{U}(1))$ граница Шилова есть окружность, а ее односвязное накрытие - прямая с обычным упорядочением. В случае $D=\mathrm{SU}(2,2) / \mathrm{S}(\mathrm{U}(2) \times \mathrm{U}(2))$ граница Шилова есть четырехмерная вещественная квадрика сигнатуры $(4,2)$. Ее односвязное накрытие, диффеоморфное $S^{3} \times \mathbb{R}$, рассматривалось Сигалом [5] как модель вселенной. Односвязное накрытие группы $G$ в этом случае есть не что иное, как группа конформных преобразований этой модели. Сигал [5] также доказал существование инвариантного упорядочения на односвязном накрытии $G / P$ для случая $G=\mathrm{SU}(n, n)$.

Используя результат Сигала, Пэнейц [6] доказал существование инвариантного упорядочения на односвязном накрытии пространства $G / P$ для произвольной эрмитовой классической простой группы Ли $G$ трубчатого типа. Из этого факта он вывел существование инвариантного упорядочения на односвязной накрывающей группы $G$. В настоящей статье проделано обратное: из существования инвариантного упорядочения на односвязной накрывающей группы $G$ выведено существование инвариантного упорядочения на односвязном накрытии пространства $G / P$. Приведенное ниже доказательство является общим; в частности, оно пригодно для группы $G$ типа $\mathrm{E}_{7}$ (единственной особой эрмитовой простой группы Ли трубчатого типа).

Инвариантным упорядочением на группе $G$ называется упорядочение, инвариантное относительно левых и правых сдвигов. Инвариантное упорядочение однозначно определяется множеством $S=\{g \in G: g \geqslant e\}$. Множество $S$ является полугруппой, инвариантной относительно внутренних автоморфизмов. Кроме того, $S \cap S^{-1}=\{e\}$. Инвариантное упорядочение на группе Ли $G$ называется непрерывным, если полугруппа $S$ замкнута и топологически порождается любой своей окрестностью единицы.

$\mathrm{C}$ каждым непрерывным инвариантным упорядочением на группе Ли $G$ связан инвариантный строго выпуклый конус $C(S)$ в касательной алгебре Ли $\mathfrak{g}$, состоящий из всех векторов, касательных к кривым, лежащим в $S$. При этом 
$\exp C(S) \subset S$, отображение $\operatorname{exp:~} C(S) \rightarrow S$ является открытым в нуле и полугруппа $S$ алгебраически порождается множеством $\exp C(S)$ [2]. Произвольный инвариантный строго выпуклый конус $C \in \mathfrak{g}$ называется допустимым, если существует такая полугруппа $S \subset G$, что $C=C(S)$.

Пусть $H-$ произвольная связная подгруппа Ли группы $G$ и $\varphi: G \rightarrow G / H$ - каноническое отображение. Инвариантное упорядочение на однородном пространстве $G / H$ называется непрерывным, если множество $R=\{x \in G / H \mid x \geqslant$ $e H\}$ замкнуто и полугруппа $\varphi^{-1}(R)$ порождается любой своей окрестностью единицы. Непрерывное инвариантное упорядочение на однородном пространстве $G / H$ называется групповым, если существует такое непрерывное инвариантное упорядочение на группе $G$ с определяющей полугруппой $S$, что $x \geqslant y$ для $x, y \in G / H$ тогда и только тогда, когда существует такой элемент $s \in S$, что $x=s y$.

Предложение. Для того чтобы непрерывное инвариантное упорядочение на группе $G$, определенное полугруппой $S$, задавало инвариантное упорядочение на $G / H$, необходимо и достаточно выполнение следующего условия: если $s, r \in S, s r \in H$, mo $s, r \in H$.

Доказательство. Пусть групповое упорядочение на $G / H$ определено корректно. Допустим, что $e \neq s \notin H$ и $s r \in H, r \in S$. Тогда $e H=s r H \geqslant s H>e H$ - противоречие. С другой стороны, пусть групповое упорядочение не определено корректно. Это означает, что не выполнено условие антисимметричности, т. е. возможен случай $a>b$ и $b>a$. Можно считать, что $b=e H$, так как упорядочение инвариантно относительно левых и правых сдвигов. Имеем $a=r b$, $b=s a, s, r \in S, s, r \notin H$. Тогда $b=s r b$ и $s r \in H$.

Если $H$ - такая подгруппа Ли, что $\operatorname{Ad}(H)$ - редуктивная линейная группа Ли, то условие в предложении эквивалентно тому, что $H \cap S=\{e\}$ [3].

В настоящей статье получены следующие результаты.

Теорема 1. Пусть $G$ - односвязная эрмитова простая группа Ли трубчатого типа, а $P$ - ее максимальная параболическая подгруппа, унипотентный радикал которой абелев. Тогда на однородном пространстве $G / P$ существует ровно одна пара противоположных непрерывных инвариантных упорядочений. Эти упорядочения являются групповыми.

Теорема 2. В условиях теоремы 1 пусть $P_{1}$ - некоторая параболическая подгруппа группь $G$, и пусть на $G / P_{1}$ существует групповое инвариантное упорядочение. Тогда подгруппа $P_{1}$ сопряжена некоторой подгруппе группы $P$.

\section{§2. Предварительные замечания}

Пусть $\mathfrak{g}$ - эрмитова простая алгебра Ли, и пусть $\mathfrak{g}=\mathfrak{k} \oplus \mathfrak{m}-$ ее разложение Картана (k - максимальная ad-компактная подалгебра). Центр $\mathfrak{z}(\mathfrak{k})$ алгебры $\mathfrak{k}$ одномерен. Правильной инволюцией алгебры $\mathfrak{g}$ называется инволюция $\sigma$ со следующими свойствами: $\sigma(\mathfrak{k})=\mathfrak{k}, \sigma(\mathfrak{m})=\mathfrak{m},\left.\sigma\right|_{\mathfrak{z}(\mathfrak{k})}=-1$. Алгебра $\mathfrak{g}$ имеет разложение $\mathfrak{g}=\mathfrak{g}^{0} \oplus \mathfrak{g}^{1}$, где $\left.\sigma\right|_{\mathfrak{g}^{0}}=1,\left.\sigma\right|_{\mathfrak{g}^{1}}=-1$. С каждой правильной инволюцией $\sigma$ алгебры $\mathfrak{g}$ можно связать вещественную форму $\mathfrak{l}=\mathfrak{g}^{0} \oplus i \mathfrak{g}^{1}$ алгебры $\mathfrak{g}_{\mathbb{C}} ;$ при этом $i \mathfrak{z}(\mathfrak{k}) \subset \mathfrak{l}$.

В пространстве $\mathfrak{m}$ существует комплексная структура, инвариантная относительно k. Она может быть определена по формуле

$$
\text { Iy }=[k, y], \quad y \in \mathfrak{m},
$$


где $k$ - подходящий элемент из $\mathfrak{z}(\mathfrak{k})$. Собственные значения оператора $\operatorname{ad}(k)$ суть $0, \pm i$.

Теперь пусть алгебра $\mathfrak{g}$ является трубчатой. Существует такая правильная инволюция $\sigma$, что алгебра $\mathfrak{l}$, определенная в первом абзаце, изоморфна алгебре $\mathfrak{g}[2]$. Пусть $\mathfrak{l}=\mathfrak{k}_{1} \oplus \mathfrak{m}_{1}-$ картановское разложение алгебры $\mathfrak{l}$. Выберем элемент $k_{1} \in \mathfrak{k}_{1}$, задающий комплексную структуру на $\mathfrak{m}_{1}$. Тогда элемент $i k_{1}$ лежит в $\mathfrak{m}$. Обозначим его через $m$. Очевидно, что оператор $\operatorname{ad}(m)$ имеет собственные значения $0, \pm 1$.

Алгебра $\mathfrak{g}$ разлагается на собственные подпространства оператора $\operatorname{ad}(m)$ :

$$
\mathfrak{g}=\mathfrak{g}_{-1} \oplus \mathfrak{g}_{0} \oplus \mathfrak{g}_{1},
$$

причем подалгебра $\mathfrak{g}_{0}$ сопряжена подалгебре $\mathfrak{g}^{0}$, определенной выше, а $\mathfrak{g}_{-1}$ и $\mathfrak{g}_{1}$ - коммутативные унипотентные подалгебры Ли. Алгебра $\mathfrak{p}=\mathfrak{g}_{0} \oplus \mathfrak{g}_{1}$ является максимальной параболической подалгеброй алгебры $\mathfrak{g}$. Ее унипотентный радикал $\mathfrak{g}_{1}$ абелев. Все максимальные параболические подалгебры алгебры $\mathfrak{g}$, унипотентные радикалы которых абелевы, сопряжены.

Пример. Рассмотрим алгебру Ли $\mathfrak{g}=\mathfrak{s u}(n, n)$ комплексных матриц порядка $2 n$ с нулевым следом, косоэрмитовых относительно эрмитовой формы с матрицей $\left(\begin{array}{cc}0 & E \\ E & 0\end{array}\right)$. Тогда $\mathfrak{g}=\left\{\left(\begin{array}{cc}A & B \\ C & -A^{*}\end{array}\right), A, B, C \in \mathfrak{g l}_{n}(\mathbb{C}), B^{*}=B, C^{*}=C\right\}$. Автоморфизм $\sigma$ есть сопряжение посредством матрицы $\left(\begin{array}{cc}E & 0 \\ 0 & -E\end{array}\right)$. Элемент $m$ представлен матрицей $\left(\begin{array}{cc}\frac{1}{2} E & 0 \\ 0 & -\frac{1}{2} E\end{array}\right)$. Следовательно, $\mathfrak{g}_{0}=\left\{\left(\begin{array}{cc}A & 0 \\ 0 & -A^{*}\end{array}\right)\right\}$, $\mathfrak{g}_{1}=\left\{\left(\begin{array}{cc}0 & B \\ 0 & 0\end{array}\right)\right\}, \mathfrak{g}_{-1}=\left\{\left(\begin{array}{ll}0 & 0 \\ C & 0\end{array}\right)\right\}$.

Пусть $G$ - односвязная эрмитова простая группа Ли с касательной алгеброй $\mathfrak{g}$. Обозначим через $G_{-1}, G_{0}, G_{1}, P$ связные подгруппы группы $G$ с касательными алгебрами $\mathfrak{g}_{-1}, \mathfrak{g}_{0}, \mathfrak{g}_{1}, \mathfrak{p}$ соответственно. Очевидно, что $G_{-1}=$ $\exp \mathfrak{g}_{-1}, G_{1}=\exp \mathfrak{g}_{1}$. Имеется разложение $P=G_{0} G_{1}$, где $G_{0}$ - подгруппа Леви, а $G_{1}$ - унипотентный радикал. Подгруппа $P$ - максимальная параболическая подгруппа, имеющая абелев унипотентный радикал. Все максимальные параболические подгруппы группы $G$, радикалы которых абелевы, сопряжены подгруппе $P$.

\section{§3. Доказательство теоремы 1}

Пусть на односвязной эрмитовой простой группе $G$ задано непрерывное инвариантное упорядочение с определяющей полугруппой $S$. На симметрическом пространстве $G / G_{0}$ существуют две пары противоположных непрерывных инвариантных упорядочений [2]; из них одна пара состоит из групповых упорядочений [3]. Любое непрерывное инвариантное упорядочение на группе $G$ индуцирует упорядочение на $G / G_{0}$. Так как $\operatorname{Ad}\left(G_{0}\right)$ - редуктивная линейная группа Ли, то отсюда следует, что $G_{0} \cap S=\{e\}$.

Рассмотрим пересечение подгруппы $P=G_{0} G_{1}$ с полугруппой $S$.

Лемма 3. $S \cap P=S \cap G_{1}$. 
Доказательство. Рассмотрим элемент $g \in S \cap P, g=g_{0} g_{1}, g_{0} \in G_{0}, g_{1} \in G_{1}$. Предположим, что $g_{0} \neq e$. Элемент $g_{1}$ можно представить в виде $g_{1}=\exp \xi$, $\xi \in \mathfrak{g}_{1}$. Тогда для любого $u \in \mathbb{R}$

$$
\operatorname{Ad}(\exp u m) g=g_{0} \operatorname{Ad}(\exp u m) g_{1}=g_{0} \exp \left(e_{u} \xi\right) \in S .
$$

Устремляя параметр $u$ к $-\infty$ и пользуясь замкнутостью полугруппы $S$, получим, что $g_{0} \in S,-$ противоречие, так как $S \cap G_{0}=\{e\}$.

Кусочно-дифференцируемую кривую $g(t)$ на группе $G$ назовем допустимой, если $g^{\prime}(t) \in g(t) C(S)$ для любого $t$. Множество всех элементов $g \in G$, для которых существует допустимая кривая, соединяющая $e$ с $g$, совпадает с $S$ [2].

Дифференцируемую функцию $\varphi$ на группе $G$ назовем возрастающей, если она непостоянна и $\mathrm{d}_{g} \varphi \geqslant 0$ на $g C$ для любого $g \in G$. Множество возрастающих функций инвариантно относительно левых и правых сдвигов. Возрастающая функция возрастает (быть может, не строго) вдоль любой допустимой кривой. Возрастающая функция существует для любого допустимого конуса $C$ [4].

Сдвинув функцию $\varphi$, можно добиться того, чтобы $\mathrm{d}_{e} \varphi \neq 0$. Далее, с помощью внутренних автоморфизмов можно получить из $\varphi$ возрастающие функции $\varphi_{1}, \ldots, \varphi_{n}$, образующие систему координат в некоторой окрестности $O(e)$ единицы группы $G$. Будем считать для определенности, что $\varphi_{i}(e)=0$ при всех $i$ и что $O(e)$ в координатах $\varphi_{1}, \ldots, \varphi_{n}$ есть шар радиуса 1 с центром в начале координат. Через $O_{r}(e)$ будем обозначать шар радиуса $r$ с центром в начале координат.

Пусть $g \in S \cap G_{1}$ и $g(t)$ - допустимая кривая, соединяющая e с $g$.

Лемма 4. $g(t) \subset G_{1}$.

Доказательство. Имеем $g=\exp \xi, \xi \in \mathfrak{g}_{1}$. Так как $\operatorname{Ad}(\exp u m) \xi=e^{u} \xi$, то для любого $r<1$ найдется такое $u \in \mathbb{R}$, что $\operatorname{Ad}(\exp u m) g \in O_{r}(e)$. Тогда и $\operatorname{Ad}(\exp u m) g(t) \in O_{r}(e)$, так как $\varphi_{i}$ не убывает на $g(t)$. Таким образом, при $u \rightarrow-\infty$ кривая $\operatorname{Ad}(\exp u m) g(t)$ стягивается в $e$. Оператор $\operatorname{Ad}(\exp u m)$ действует на подгруппе $G_{0}$ тождественным преобразованием, а на подгруппе $G_{-1}=\exp \mathfrak{g}_{-1}$ следующим образом: $\operatorname{Ad}(\exp u m) \exp \eta=\exp \left(e^{-u} \eta\right)$. Значит, кривая $g(t)$ целиком лежит в $G_{1}$.

Теперь пусть $s, r \in S, s r \in G_{1}$, и пусть $s(t), r(t), t \in[0,1],-$ допустимые кривые, соединяющие $e$ с элементами $s$ и $r$ соответственно. Рассмотрим следующую допустимую кривую $q(t): q(t)=s(2 t)$ при $t \in[0,1 / 2], q(t)=\operatorname{sr}(2 t-1)$ при $t \in[1 / 2,1]$. Эта допустимая кривая соединяет $e$ с $s r$. По лемме 4 она целиком лежит в $G_{1}$. Значит, $s, r \in G_{1}$. Следовательно, полугруппа $S$ индуцирует групповое упорядочение на $G / P$.

Замечание. Приведенное доказательство существования группового инвариантного упорядочения справедливо не только для $P=G_{0} G_{1}$, но и для любых групп вида $H G_{1}$, где $H \subset G_{0}$ - связная подгруппа Ли.

Теперь докажем, что непрерывное инвариантное упорядочение на $G / P$ единственно.

Каждому инвариантному упорядочению на $G / P$ соответствует $\operatorname{Ad}(P)$-инвариантный замкнутый строго выпуклый конус в пространстве $\mathfrak{g} / \mathfrak{p}$, которое можно отождествить с пространством $\mathfrak{g}_{-1}$. Этот конус состоит из всех векторов, касательных к кривым, лежащим в $R$, где $R=\{x \in G / P \mid x \geqslant e P\}$. Упорядочение однозначно восстанавливается по $R[2]$. 
Лемма 5. $\operatorname{Ad}\left(G_{0}\right)$-инвариантный конус $D$ в пространстве $\mathfrak{g}_{-1}$ единствен с точностъю до умножения на -1 .

Доказательство. Подгруппа $P_{0}=G_{0} G_{-1}$ является максимальной параболической и сопряжена подгруппе $P$. Более того, существует такой внутренний автоморфизм $\theta$, что $\theta\left(G_{0}\right)=G_{0}, \theta\left(G_{-1}\right)=G_{1}$. Тогда если $D$ есть $\operatorname{Ad}\left(G_{0}\right)$ инвариантный конус в $\mathfrak{g}_{-1}$, то $\theta(D)$ - это $\operatorname{Ad}\left(G_{0}\right)$-инвариантный конус в $\mathfrak{g}_{1}$. Следовательно, по конусу $D$ в пространстве $\mathfrak{g}_{-1} \oplus \mathfrak{g}_{1}$ можно построить четыре $\operatorname{Ad}\left(G_{0}\right)$-инвариантных конуса $\pm D \pm \theta(D)$. Проекция любого из них на $\mathfrak{g}_{-1}$ есть $\pm D$. Но (см. [2]) в пространстве $\mathfrak{g}_{-1} \oplus \mathfrak{g}_{1}$ существует ровно четыре различных $\operatorname{Ad}\left(G_{0}\right)$-инвариантных конуса с непустой внутренностью. Следовательно, конус $D$ определен с точностью до умножения на -1 .

Этот конус можно представить в явном виде. Существует взаимно однозначное соответствие между однородными пространствами типа $G / P$ и простыми евклидовыми йордановыми алгебрами [7, с. 213]. При этом соответствии $\mathfrak{g}_{-1}$ можно представить в виде $i \mathscr{J}$ для некоторой йордановой алгебры $\mathscr{J}$, a $G_{0}$ - группа линейных преобразований алгебры $\mathscr{J}$, сохраняющая конус квадратов $\Omega$. В этих обозначениях $\operatorname{Ad}\left(G_{0}\right)$-инвариантный конус в пространстве $\mathfrak{g}_{-1}$ есть $i \Omega$.

\section{§4. Доказательство теоремы 2}

Пусть снова на односвязной эрмитовой простой группе $G$ задано непрерывное инвариантное упорядочение с определяющей полугруппой $S$. Касательный конус полугруппы $S$ обозначим через $C$.

Пусть $\mathfrak{a}$ - максимальная $\mathbb{R}$-диагонализуемая подалгебра алгебры $\mathfrak{g}$, лежащая в $\mathfrak{m}$. Обозначим через $\Delta$ систему корней алгебры $\mathfrak{g}$ относительно $\mathfrak{a}$ и через $\mathfrak{g}_{\varepsilon}-$ корневое подпространство, отвечающее корню $\varepsilon$. Известно [1], что $\Delta$ есть система корней типа $C_{n}$. Пусть $\varepsilon_{1}-$ старший корень системы $\Delta$. Тогда пространство $\mathfrak{g}_{\varepsilon_{1}}$ одномерно и его базисный вектор $\alpha_{1}$ является старшим корневым вектором алгебры $\mathfrak{g}$ [1]. Таким образом, $\mathfrak{g}_{\varepsilon_{1}} \cap C \neq\{0\}[1]$. Пусть $\varepsilon_{1}, \ldots, \varepsilon_{n}$ - канонический базис системы $\Delta$ (базис, в котором система $\Delta$ имеет вид $\left.\left\{ \pm \varepsilon_{i} \pm \varepsilon_{j}, \pm 2 \varepsilon_{i}\right\}\right)$, а $e_{1}, \ldots, e_{n}$ - соответствующий двойственный базис алгебры a. Элементы $\pm e_{i}$ попарно сопряжены.

Пусть $\mathfrak{p}_{1}$ - некоторая параболическая подалгебра алгебры $\mathfrak{g}$. Тогда найдутся такие максимальная $\mathbb{R}$-диагонализуемая подалгебра $\mathfrak{a}$ и элемент $a \in \mathfrak{a}$, что $\mathfrak{p}=\bigoplus_{t \geqslant 0} \mathfrak{g}_{t}$, где $\mathfrak{g}_{t}-$ собственное подпространство оператора ad $a$, отвечающее собственному значению $t$. Максимальная параболическая подалгебра $\mathfrak{p}$ с абелевым унипотентным радикалом в обозначениях предыдущего абзаца отвечает элементу $e_{1}+\cdots+e_{n}$.

Пусть $P_{1}$ и $G_{0}$ - связные подгруппы группы $G$ с касательными алгебрами $\mathfrak{p}_{1}$ и $\mathfrak{g}_{0}$ соответственно. Тогда $\mathrm{Ad} G_{0}$ - редуктивная линейная группа Ли.

Пусть $a=\sum a_{i} e_{i}$. Можно считать, что все $a_{i}$ неотрицательные (в противном случае заменим элемент $a$ на сопряженный). Если все $a_{i}$ положительны, то $\mathfrak{p}_{1} \subset \mathfrak{p}$. Если же $a_{k}=0$, то в подалгебре $\mathfrak{g}_{0}$ содержится подпространство $\mathfrak{g}_{\varepsilon_{k}}$, сопряженное пространству $\mathfrak{g}_{\varepsilon_{1}}$, и, следовательно, $\mathfrak{g}_{0} \cap C \neq\{0\}$. В последнем случае $G_{0}$ пересекается с $S$ по внутренней точке [3], а значит, и подгруппа $P_{1}$ пересекается с $S$ по внутренней точке; следовательно, на $G / P_{1}$ не существует группового инвариантного упорядочения [3]. 


\section{ЛитеРАТУРА}

[1] Э. Б. Винберг, Инвариантные выпуклые конусы и упорядочения в группах Ли, Функц. анализ и его прил., 14:1 (1980), 1-13.

[2] Г. И. Ольшанский, Выпуклые конусы в симметрических алгебрах Ли, полугруппъ Ли и инвариантные причинные структуры (упорядочения) на псевдоримановых симметрических пространствах, ДАН СССР, 265:3 (1982), 537-541.

[3] А. Л. Константинов, Инвариантные упорядочения в однородных пространствах простых групn Ли, Вестн. Моск. ун-та, сер. 1, матем., мех., 2006, № 6 .

[4] Г. И. Ольшанский, Инвариантные упорядочения в простых группах Ли. Решение задачи Э. Б. Винберга, Функц. анализ и его прил., 16:4 (1982), 80-81.

[5] I. E. Segal, Mathematical Cosmology and Extragalactic Astronomy, Academic Press, New York, 1976.

[6] S. Paneitz, Invariant convex cones and causality in semisimple Lie algebras and groups, J. Funct. Anal, 43 (1981), 313-359.

[7] J. Faraut, A. Koranyi, Analysis on Symmetric Cones, Oxford Mathematical Monographs, Oxford University Press, 1994.

Московский государственный университет

e-mail: lelik_msu@rambler.ru

Поступило в редакцию

7 сентября 2006 г. 